\title{
Psychedelic compound in ecstasy moves closer to approval to treat PTSD
}

\section{A promising treatment that uses MDMA could help people suffering with post-traumatic stress disorder.}

\section{Amy Maxmen}

28 April 2017

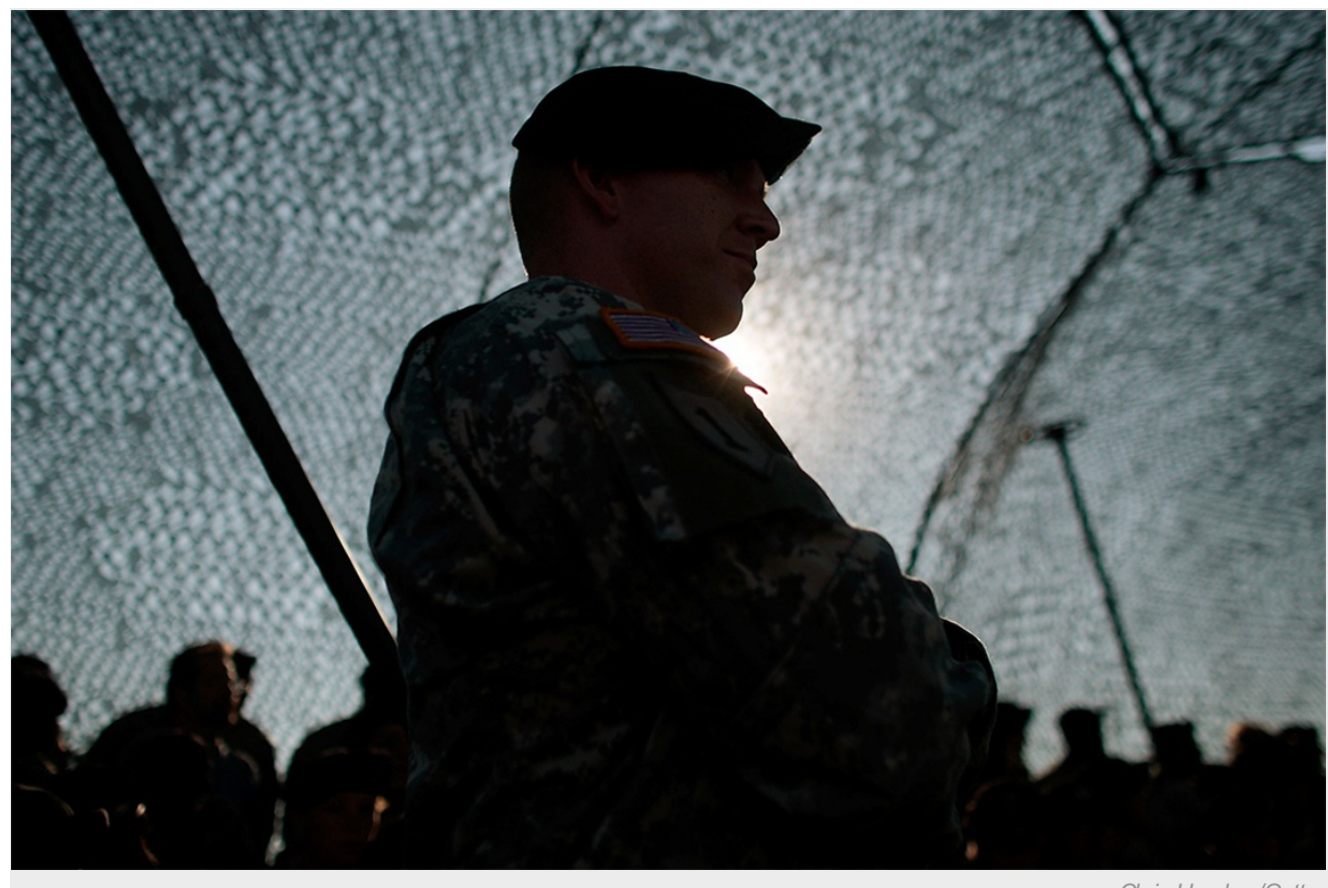

Chris Hondros/Getty

Psychedelic drugs could soon help people, including soldiers, who suffer from post-traumatic stress disorder with the pain of recalling traumatic memories.

Psychologists have occasionally given people psychedelic drugs such as LSD or magic mushrooms to induce altered states, in an attempt to treat mental illness. Today, many of those drugs are illegal, but if clinical trials testing their efficacy yield positive results, a handful could become prescription medicines in the next decade. The furthest along in this process is MDMA — a drug sold illegally as ecstasy or Molly — which is showing promise in the treatment of post-traumatic stress disorder (PTSD).

Last week, at the Psychedelic Science 2017 conference in Oakland, California, researchers presented unpublished results from phase II trials involving a total of 107 people diagnosed with PTSD. The trial treatment involved a combination of psychotherapy and MDMA (3,4-methylenedioxymethamphetamine). The US Food and Drug Administration (FDA) reviewed these data in November, which were not released to the public at the time. The agency recommended that the researchers move forward with phase III trials, the final stage before potential approval of the drug.

At the conference, researchers affiliated with the non-profit organization that is sponsoring the trials, the Multidisciplinary Association for Psychedelic Studies (MAPS) in Santa Cruz, California, presented some of their latest resutls. They used a cinically validated scale that assesses PTSD symptoms such as frequency of nightmares and anxiety levels. More than one year after two or three sessions of MDMA-assisted therapy, about $67 \%$ of participants no longer had the illness, according to that scale. About $23 \%$ of the control group — who received psychotherapy and a placebo drug — experienced the same benefit.

Researchers leading those trials are now training a cadre of therapists to deliver a form of psychotherapy tailored for use with MDMA, in preparation for the phase III trials. They consider this component essential. "I've seen people in my practice who took MDMA at a party and weren't prepared for the memories that came up, and it was really harmful for them," says Michael Mithoefer, a psychiatrist in Charleston, South Carolina, and a principle investigator in the MDMA trials. 
to treat PTSD fail to respond to therapies including serotonin-reuptake inhibitors - a class of drugs often used as antidepressants and cognitive behavioural therapy.

"The results l've seen so far with MDMA are so much better than anything l've seen so far," Mithoefer says.

\section{Therape utic sweet spot}

PTSD treatment commonly involves getting a patient to repeatedly recall traumatic events, to extinguish the fear associated with the experience ${ }^{1}$. But that method can fail if patients can't describe the memory because they have walled it off. Other people have such a charged emotional response that the recollection causes harm. "Because of this stress, many patients will drop out of treatment," explains Daniel Zuj, a research psychologist at the University of Tasmania in Launceston, Australia, who is not involved in the MDMA trials.

In the 1990s, scientists demonstrated in rodents and humans that MDMA was reasonably safe when taken a few times in a controlled setting. The FDA permitted researchers to move forward with clinical trials exploring the drug as a treatment for PTSD.

Studies suggest that MDMA reduces the fear response ${ }^{1}$, and triggers the release of serotonin and other neurotransmitters that induce a feeling of well-being ${ }^{2}$. In this way, individuals may recall events multiple times, in detail, without panicking. "MDMA provides a sweet spot where therapeutic change can happen," says Mithoefer. "It affects neural networks so that people's experiences are not hijacked by fear."

\section{Sustained results}

Investigators with MAPS hope to enrol up to 300 people with PTSD to participate in the upcoming phase III trials. The researchers will spend this year training therapists from 14 clinics across North America and Israel to deliver the MDMA-assisted psychotherapy.

They developed their approach by combining modern PTSD therapy with techniques used by LSD researchers in the 1960s. Two therapists supervise the patient while he or she is under the influence of the drug; this lasts five to eight hours for MDMA. It's essential that people feel they are in a safe, comfortable setting with someone who can oversee the experience, says Alicia Danforth, a clinical psychologist at the Harbor-UCLA Medical Center in Torrance, California, who is involved in MAPS-sponsored trials studying MDMA's effects on social anxiety in adults with autism.

During his conference presentation, Mithoefer played a video of a former US marine under the influence of MDMA recounting the time his military jeep exploded during a tour in Iraq. The soldier, positioned on a narrow bed between Mithoefer and his wife Annie, a psychiatric nurse, describes the panic that accompanies his memories. But then, he says, an inner-voice assures him that he'll be all right. "I feel things come up and then blow away like sand," the marine says. Michael Mithoefer says it's been five years since the marine's session. "We are still in touch," he says, "and that effect has lasted."

Nature | doi:10.1038/nature.2017.21917

\section{References}

1. Kilpatrick, D. et al. J. Trauma Stress 26, 537-547 (2013).

2. Vollenweider, F. X., Gamma, A., Liechti, M. \& Huber, T. Neuropsychopharmacology 19, 241-251 (1998). 